\title{
BIOASTRONOMY (REPORT OF IAU COMMISSION 51)
}

\author{
Marx, George \\ Department of Atomic Physics, Eötvös University \\ Budapest H-1088
}

\begin{abstract}
In the recent years, science have become able to give more definite answers to the questions of the abundance of planetary systems, of the pathways of chemical evolution leading to the emergence of simple self-reproducing structures, to the origins of life within and beyond the Solar System. At the same time a new question has been raised: the duration of life-sustaining environment, what is a prerequisit for the emergence of technology. Thanks to the fast progress of computer technology, the search for intelligent radio signals has become affordable. The number of observatories participating in this venture increases rapidly. Preparations are in progress for a large scale systematic survey.
\end{abstract}

\section{The Beginnings}

The first professional watch looking for intelligent extraterrestrial radio signals was performed just 30 years ago (Drake 1960), and in three decades not only the interest, but the professional scientific and technical activity increased exponentially. Since 1979, the bioastronomy sessions attracted special interest at each General Assembly. Reflecting these developments, the Commission 51 on Bioastronomy was established at the General Assembly of IAU in 1982, at the iniciative of Michael Papagiannis. Michael Papagiannis (1982-1985), Francis Drake (1985-1988), George Marx (1988-1991) served as first presidents of the Commission. The members of the Commission 51 were reached by the Bioastronomy News, edited by Michael Papagiannis. The highlights of the activity were the Bioastronomy Symposia in Boston (USA, 1984), Balaton (Hungary, 1987), Val Cenis (France, 1990). The interest of astronomers, physicists, chemists, climatologists, biologists, psychologists, computer scientists and engineers has been concentrated upon the goal: to understand man's place in the Universe. While the mosaics begin to fit together, it is hard to suppress the philosophical, sociological, ethical emotions in the depth of our mind, raised by discussing these interwoven interdisciplinary connections. The attention of the Commission members certainly focuses onto the scientific aspects of planetary evolution and the technological search for extraterrestrial intelligence.

\section{The Origins of Free Energy}

The early Universe was hot and dense, with abundant collisions of its constituents, therefore it was in thermal equilibrium, as indicated by the relic black body radiation: it shows an isotropy up to five decimals and a Planck shape up to two decimals. Consequently the free energy in the Early Universe was zero. The gravitational instability resulted in expansion, the expansion caused adiabatic cooling. Its different (massless and massive) constituents - having different scaling properties - cooled at different rates. The thermodynamic (thermal, density, chemical) differences tried to level up irreversibely (creating considerable entropy in the Universe, observable today mainly as microwave radiation background), but in cooler and thinner times the coupling among constituents weakened, 
thus the differences temporarily survived: free energy has been created. Searching for potential sites of life means looking for regions where the thermalization has been prolonged by the slowness of weak interactions (radioactivity and gravity) for billions of years, requested for the emergence and development of life and technology. (Marx 1990) Possible places are:

- the vicinity of main sequence stars (time has been offerred by the low probability of the radioactive transition ${ }^{1} \mathrm{H}+{ }^{1} \mathrm{H} \rightarrow{ }^{2} \mathrm{H}+\mathrm{e}^{+}+\nu$ at the beginning of nuclear build-up in stars), where starshine creates thermal differences on planets (e.g. on Earth);

- tidal friction on satellites of massive bodies, if the rotation and orbital excentricity decays only slowly due to gravitational resonance and other favourable circumstances (like in the case of the Jupiter-moons Io and Europe);

- radioactive heating of otherwise cool bodies by long-lived radioactive nuclei like ${ }^{26} \mathrm{Al},{ }^{40} \mathrm{~K},{ }^{235} \mathrm{U},{ }^{232} \mathrm{Th}$, created in supernove explosions (deep oceanic hot rifts on Earth, perhaps melted comets in the early Solar System, or faraway solid bodies massive enough to show volcanic activity and plate tectonics) (Corliss 1987);

- it may be, more exotic and less explored sites like objects bombarded by cosmic ray, UV light, meteors in early systems, or cooling neutron stars, or decaying black holes (Dyson 1982).

\section{Chemical Evolution}

The common wisdom supposes that if the temperature on an astronomical object is in the interiall $0^{\circ} \mathrm{C}-80^{\circ} \mathrm{C}$, if there are liquid water and compounds of carbon and nitrogen available, if there is a sufficient source of free energy present, then chemical evolution will run, leading to the emergence of self-replicating organic structures. This optimistic view is supported by the terrestrial evidence, that soon after the formation of liquid oceans upon the cooling Earth (about 3.8-3.9 billion years ago), rather developed stromatolitelike structures were deposited in the sediments, (3.6-3.8 billion years ago). This seems to indicate a spontaneous emergence of life with high probability, if the appropriate physical and chemical conditions are present.

To elaborate this empirical conclusion in more details, the possible steps of the chemical evolution have been investigated.

In interstellar clouds, and in cold envelopes of late carbon stars more than 80 different molecules of $\mathrm{H}, \mathrm{C}, \mathrm{O}, \mathrm{N}, \mathrm{S}, \mathrm{Si}, \mathrm{Cl}, \mathrm{F}, \mathrm{P}, \mathrm{Na}, \mathrm{K}, \mathrm{Al}$ were found by spectroscopy, up to chains of 6 carbon atoms, with a high abundance of $\mathrm{HCN}$ and $\mathrm{HNC}$ molecules which are inclined to form polymers (Irvine, 1989). Broad infrared bands indicate aromatic matter, too, made of 20-30 carbon atoms (Herbst, 1990). Dust particles (silicates?) of the size of $0.1 \mu \mathrm{m}$ are also present, attenuating the star light. Broad infrared absorption bands imply that the dust is covered by ice and perhaps by aromatic matter. Similar stuff might have served as building material at the formation of the Solar System. This sort of chemical composition has been confirmed, using the visits to the Halley comet (Szegö 1987, Levasseur-Requard 1988).

Experiments show that in liquid water, in the presence of a base (such as $\mathrm{NH}_{3}$ ) the $\mathrm{HCN}$ polymerizes spontaneously at room temperature, leading towards nucleotides and polypeptides, without the formation of amino acids. This kind of polymerisation has been.seen on the black Halley comet (Szegö, 1987), it could account for the dark 
materials on some asteroids, and for much of the yellow-orange-brown coloration of the atmospheres of the Jupiter, Saturn and Titan (Matthews, 1990). As carriers and catalysers of polymerisation, the silicates (clay) and iron oxides (rust) could serve, perhaps playing the role of first templates (Nussinow-Maron 1990, Arrhenius 1988).

Planet Earth is certainly a site in our Solar System where life has emerged. Most of the volatiles dissociated and escaped, when the intensive radioactivity (inherited from the supernova-ejected cloud) melted the body of Earth. This is indicated among others by the terrestrial absence of $\mathrm{Ne}$ (one of the most common elements in the outer space and in stars). The outgasing of the cooling planet produced later a $\mathrm{H}_{2} \mathrm{O}$ ocean and a $\mathrm{CO}_{2}$ atmosphere (like on Venus and Mars). The organic molecules (precursors of life) were possibly supplied by the impacts of micrometeorites, asteroids and comets in the early era of heavy bombardement 4.5-3.5 billion years ago (Maurette-Bonny 1990, Belsemme 1990). This bombardement can be made responsible for the supply of the majority of the oceanic water and atmosphere of the present Earth, enriched with the above-mentioned organics (Chiba 1990).

In order to arrive at sophisticated life forms (intelligent enough to participate in interstellar radio communication) beside a habitat convenient for the emergence of life, also time (several billion years) is needed, providing appropriate physical and chemical conditions. As we shall see, this second condition is rather restrictive, suppressing the chances of extraterrestrial intelligence considerably. The on-site search for (live or extinct) primitive life forms is of high importance. For this reason let us focus our attention to the candidate habitats in our Solar System.

\section{Search for Life in the Solar System}

The Sun was about $30 \%$ less luminous at the beginning of its main sequence phase 4.6 billion years ago than it is now. Due to the gradual depletion of its hydrogen by thermonuclear fusion, it was forced to warm gradually since that. In the future 3 billion years from now, the Sun's luminosity will increase further by about $50 \%$ of the present luminosity. For the polymerisation of simple carbonic molecules, liquid water is requested. In the absence of any thermostat, the belt of liquid water moves outward with a considerable speed in the Solar System. If there is liquid ocean on a planet the $\mathrm{CO}_{2}$ may be largely dissolved in it. One can calculate that 4 billion years ago the liquid water belt was at the distance of Venus, now it is at about the Earth, and 3 billion years from now it will reach the Mars. About 5 billion years from now, the Sun is expected to enter its red giant stage, increasing its luminosity by a factor of about 300 . At that time, liquid water will exist only on the surfaces of the present icy Galilean satellites. Ocean will persist there for about one billion years. As the Sun evolves further at an accelerating rate, Titan may enter the liquid water phase for perhaps 100 million years. As we see, the habitat with liquid water around the Sun is highly varying in time (Doyle 1990). The width of this habitat at a given time is restricted as well. In his pioneering paper, discussing the role of planetary atmosphere, Hart concluded that for a main sequence star of 1 solar mass, the habitable zone extends only from $0.95 \mathrm{AU}$ to $1.0034 \mathrm{AU}$ (Hart 1978). But in the 1980-es, especially in the last years our understanding widened this range. Let us list the possible habitats of life in the light of our new knowledge concerning climatic changes. 
Venus is presumed to have been formed with a water content comparable to (one third of) that of Earth. The $\mathrm{CO}_{2}$ was solved originally in the oceans. When the oceans evaporated due to the increasing solar luminosity, the infrared absorbtion of the thick $\mathrm{CO}_{2}$ atmosphere produced a runaway greenhouse effect. The $\mathrm{H}_{2} \mathrm{O}$ molecules were dissociated by solar UV light, subsequently the $\mathrm{H}_{2}$ escaped to the outer space within the first billion years of the history of the Solar System (Kasting 1988). Now Venus is hot, dry and lifeless.

Earth has managed to keep its atmosphere habitable through 3.5 billion years, in spite of the increasing solar luminosity. This can be explained by the interplay of plate tectonics (Kasting 1988) and the biosphere (Lovelock 1989). If the atmospheric composition 2-3 billion years ago were the same as today's, the Earth would have been frozen because of the low solar luminosity (Sagan 1972). One has to assume that the early Earth had a thin $\mathrm{CO}_{2}$ atmosphere, resulting just in appropriate infrared absorption and greenhouse warming. Later on, the increasing solar luminosity was compensated by the decrease of the $\mathrm{CO}_{2}$ content of the atmosphere. The temperature-regulation might be performed by the carbonate-silicate cycle: The atmospheric $\mathrm{CO}_{2}$ is absorbed in the ocean, where different life-forms transform $\mathrm{CO}_{2}$ to $\mathrm{Ca}$ carbonate. When the sinking sediments will be heated geothermally, $\mathrm{Ca}$ silicate is formed and $\mathrm{CO}_{2}$ is liberated. Plate tectonics and volcanism raises the silicates to the surface of continents, bringing them in contact with air. Weathering of rocks acts again as a sink of $\mathrm{CO}_{2}$ : it transforms the silicates to carbonates. The rate of weathering may be enhanced by 2 or 3 orders of magnitude by the increased organic soil surface and the organic acidity of the soil (Schwartzmann 1989). The biological activity and the land weathering are enhanced at higher temperature, they get suppressed at lower temperature, what may result in a thermostat effect on Earth, making it the only continuously habitable place in the Solar System. This steady temperature has offered a chance for the development of intelligence and technology.

Mars displays fluvial morphologies, implying the presence of running water in the past. The runoff channels were formed some 3.8 billion years ago (Squires 1989, Cabrol 1990, Grin 1990). The $\mathrm{CO}_{2}$ might have been pumped from the carbonate rocks into the atmosphere by the meteoric bombardment (Carr 1989). After 1 billion years, the atmosperic $\mathrm{CO}_{2}$ went to carbonate rocks. The body of the planet is too small, therefore the radioactive heat cannot recycle the carbon through tectonic activity. After the disappearance of the $\mathrm{CO}_{2}$ greenhouse effect the planet became frozen (Pllack 1987). This makes Mars to be a good place for search of extint primitive life forms. Over $2 / 3$ of the planet's surface is older than 3.5 billion years, thus Mars may hold records of early life forms. - In the future, 1 billion years from now, the solar luminosity will be larger by $20 \%$ than it is now, letting liquid water reappear on Mars as a consequence of greenhouse heating (Doyle 1990).

Europe, satellite of Jupiter shows a smooth ice-covered surface, with rifts indicating some thermal activity. Under the ice cover an ocean may exist as a result of tidal heating (Reynols 1987). The tidal heat arises due to the excentric orbit, what is kept excentric by the Laplacean resonance between the Jupiter-moons Io, Europe and Ganymede.

Triton and Titan share a predominantly nitrogen atmosphere with Earth. The fly-by of Voyager-1 in 1980 and the occultation with the star 28 Sagittarii in 1989 offerred more details about Titan's atmosphere. Triton and Titan show evidence of chemical evolution, but liquid water (a prerequisit for efficient polymerisation) will appear only for a short 
period when the Sun reaches the red giant state (Braic 1990).

Comets are composed of water ice, they are rich in organics. At the formation of the Solar System (4.6 billion years ago) radioisotopes were aboundant. Radioactive heating by the decay of ${ }^{26} \mathrm{Al}$ could have resulted in the formation of liquid water inside large comets $(>10 \mathrm{~km})$ on a timescale comparable to the half life of ${ }^{26} \mathrm{Al}(0.7$ million years $)$. If the cometary material is a good insulator, the cometary ocean may last up to 1 billion years (Irvine 1981). Life originated on Earth 3.6-3.8 billion years ago (Schopf 1983), therefore the cometary origin of terrestrial life cannot be completely excluded (Doyle 1990): conditions in the chemically reducing cometary ocean might be closer to the conditions of Miller's synthesis experiment, than on Earth at any time. (Even without liquid comets the shocks of cometary impacts could drive further steps of chemical evolution in the cometary organic materials.)

\section{Extrasolar Planetary Systems}

Any gravitationally bound bulk of atoms has to give off energy into its cooling cosmic environment. Loss of energy means gravitational contraction. The shrinkage to a single point is prevented by the inherited angular momentum of the cloud. This explains the considerable abundance of multiple star systems. This is why our Sun has got planets and an Oort cometary disk as well.

The IRAS infrared-sensing satellite observed several hundred times more intensive infrared emission from about hundred main sequence stars than expected from the Planckcurve extrapolation of their optical luminosity. This may be due to a dust disk having a temperature of $30-150 \mathrm{~K}$, orbiting around the star. In the case of $\alpha \mathrm{Lyr}, \beta \mathrm{Pic}, \beta \mathrm{PsA}$ this disk has been resolved (by covering the central star). More than $15 \%$ of the nearly main sequence stars show such an infrared excess, including some stars older than 2 billion years, and the presence of excess is apparently independent of whether the star is multiple or single. This fact indicates that habitats for prebiotic chemical evolution are not uncommon in our astronomical environment. The question arises whether these grain disks are young systems which gradually disappear as planets accrete? Or do they coexist with planets? Or does their presence indicate that planets have not succeeded to be formed? The answer could be obtained by observing the corresponding infrared-shining disk around the Solar System but that seems to be a very difficult task technically.

The search for big planets by observing the periodic transversal motion of stars has a long history, without definit conclusions. At present, the most promising method for finding giant planetary companions around stars is the observation of periodic changes in the radial velocity of the star from Doppler shift. In the past 9 years 21 stars have been studied. The external error is as small as $14 \mathrm{~m} \mathrm{~s}^{-1}$. In several cases, the observed periodicity may be explained by chromospheric activity (indicated by a correlation between velocity change and CaII line width). But 7 stars show long term low level velocity variations, consistent with planetary companions. One of them, the $\varepsilon$ Eridani (target of the first OSMA radio watch, Drake 1961), shows velocity variation and infrared excess as well.

The direct optical sighting of a planet is a task for the future. The planned Astrometric Imaging Telescope, to be placed onto a satellite, is intended to have a resolution of 10 microseconds of arc, it would be able to detect Uranus sized planets around hundreds of 
nearby stars (Terille 1987). It is an important achievement of the 1980-es anyway that empirical evidences quoted above support the general theoretical view: sites for chemical evolution around stars may be rather abundent in our galactic neighbourhood.

\section{Danger Zones}

It is well accepted that stars are born nowadays at the maxima of the density waves sweeping around in the Galaxy. The young stars indicate these regions as bright "spiral arms". Due to the short life of very massive stars, supernova explosions are frequent in these spiral arms. One of them probably triggered the formation of the Solar System.

A supernova explosion increases the intensity of cosmic radiation by two orders of magnitude within $10 \mathrm{pc}$. (A nearby supernova would add $40 \mathrm{mSv} / \mathrm{yr}$ radiation dose to the natural background of $2 \mathrm{mSv} / \mathrm{yr}$ on Earth.) This would increase the lethal cancer and genetic risk, thus getting into a spiral arm would endanger the life in the planetary system. It may be that the time offerred to life in a planetary system is the time spent between spiral arms (Marochnik 1987). Our Sun performs Kepler orbit around the centre of the Galaxy, and it happens, that the angular velocity of this Kepler orbit $(25 \mathrm{~km} / \mathrm{s}$ per $\mathrm{kpc})$ does not differ significantly from the angular velocity of the galactic density wave $(23.6+3.6$ $\mathrm{km} / \mathrm{s}$ per $\mathrm{kpc}$ ). This means that our Sun has an orbiting period of 0.2 billion years in the Galaxy, but it can spend billions of years, after having left a spiral arm (its birth place) and before entering an other spiral arm (wiping out life in the Solar System). By accepting this conclusions, one may argue that the search for continuosly habitable zones (technological civilizations) should be concentrated to this restrieted corotation zone, which might be the Galactic Belt of Life (Marochnik 1987, Balázs 1987, Suchkov 1990).

Under fortunate conditions, the intensive cosmic radiation may be a driving force of chemical evolution, responsible perhaps for the very origin of life even at places far from stars (Suchkov 1990). It seems, however, to be more probable, that enhanced cosmic radiation, produced by a nearby supernova explosion, might cause mass extinction (of dinosaurus, e.g.), due to cancers and mutations. There are overwhelming evidences of mass extinctions in the geological history of Earth. Beside supernovas, several other possible cosmic causes of biological mass extinctions were suggested, e.g. passing through cosmic cloud, or massive bombardment by asteroids and comets (Alvarez 1980, 1987). The later view is based on enhanced geological Ir abundance in the "danger layers". Ir is a heavy element, which sunk to the core of Earth when it was melted by the early intensive radioactivity. Ir is more abundant now in asteroids where separation by weight has not occurred. If the Ir rich geological layers are separated by regular time intervals, the periodic bombardment might have deeper astronomical reason (e.g. orbit of the Sun in the Galaxy, or an orbiting Death Star around the Sun with a very high excentricity, Muller 1985).

All these arguments are very problematic. If there is, however, a regular astronomical explanation behind terrestrial extinctions, the same reason might serve also as the driving force for biological evolution, by weakeming the conservative ruling species. Both may have valuable restrictions, when looking for possible sights of intelligent technology. (See the special session at the IAU General Assembly in 1991.) 


\section{Search for Extraterrestrial Technology}

The paper of historical importance, written by Giuseppe Cocconi and Phil Morrison (1959), called the attention to the possibility of radio messages of extraterrestrial origin. In the decades since then a huge number of observations tried to catch these potential messages in the $\mathrm{MHz}-\mathrm{GHz}$ radio band or elsewhere. Big diskes scanned the sky on four continents, tuned to notable frequencies. Unfortunately, observation time costs money, therefore most observations run only for a limited period, looked for selected frequencies at selected targets. But "the absence of evidence is not an evidence of absence" (Sagan).

The world's oldest continuing Search for Extraterrestrial Intelligence is the Ohio State University SETI program, routinely searching through the entire sky for signals. With equipment advances, the Ohio State University telescope is now able to track an object for hours. When the computers find something, it zooms in automatically for more detailed in*estigation.

The Planetary Society's SETI program, using the Megachannel Extraterrestrial Assay, extends its activity to the southern sky (META II) in the Argentine Institute of Radio Astronomy.

In the next chapter, a full cataloge of observations, performed so far is printed. The list has been collected and updated by Jill Tarter, who can offer more detailed information to those who are interested.

The most ambitious microwave observing project for extraterrestrial technology will be NASA's program, a cooperation between the Life Science Division at the NASA Headquarter, the NASA's Ames Research Centre and the NASA's Jet Propulsion Laboratory. A 6 year long development phase has been completed. Microwave frequencies between 1 and $10 \mathrm{GHz}$ are recommended where the galactic and atmospheric noise is the lowest. The sophisticated signal processing system will be connected to the NASA Deep Space Network antennas and the largest existing telescopes. The Targeted Search will examine 800 sun-like stars within $30 \mathrm{pc}$, with a frequency resolution of $1 \mathrm{~Hz}$. The Sky Survey will scan the entire sky with a frequency resolution of $30 \mathrm{~Hz}$. If the funds will be granted, the six years long observation will start on 12 October 1992 - the 500th anniversary of Columbus' discovery of the New World on our planet.

At the 3rd Bioastronomy Symposium the IAU Commission 51 decided to organize a SETI Global Network in cooperation with the SETI Committee of the International Academy of Astronautics, in order to coordinate the activities of potential discoverers through fast direct exchange of information among the institutions of the 10 nations, involved in the research (Heidmann 1990). Jean Heidmann (Paris Observatory, Mendon), has been charged to organize the SETI Global Network for the International Space Year 1992.

And what to do, how to react, if an intelligent message will be received, and the signal will be confirmed by independent observatories? This question has been discussed repeatedly at several meetings, last time at the 3rd Bioastronomy Symposium (Almár 1990). A Declaration of Principles, concerning activities following the detection of extraterrestrial intelligence, has been accepted by the International Academy of Astronautics and IAU Commission 51. The full text of the Declaration is reprinted here. 


\section{References}

Bioastronomy 1, IAU Symposium 112 (1984): "The Search for Extraterrestrial Life: Recent Developments," edited by M.D. Papagiannis. Reidel, 579 pages.

Bioastronomy 2, IAU Colloquim 99 (1987): "Bioastronomy: The Next Steps", edited by George Marx. Kluwer Academic, 434 pages.

Bioastronomy 3 (1990):" Bioastronomy: the Exploration Broadens," edited by J. HeidmanM. Klein. Springer Lecture Series.

Almár, $I$ (1990) in Bioastronomy 3 proceedings

Alvarez, L.W. et al (1980) Science 208, 1095.

Alvarez, L.W. (1986) Physics Today 40, 24-33.

Backman,D. (1990) in Bioastronomy 3 proceedings

Backman,D. -Parese, $F$ (1991) in "Protostars and Planets III", University of Arizona Press Balázs, B. (1987) in Bioastronomy 2 proceedings 61-66.

Braic, A. et al (1990) in Bioastronomy 3 proceedins

Cabrol, N.A. (1990) in Bioastronomy 3 proceedings

Campbell, B. et al (1990) in Bioastronomy 3 proceedings

Carr, M.H. (1989) Icarus 79, 311-327.

Chiba, C.F. (1990) Nature 949, 129-133.

Cocconi, G.-Morisson, P. (1969) Nature 184, 844-849.

Corliss, J.B. (1987) in Bioastronomy 2 proceedings 195-200.

Delsemme, A.H. (1990) in Bioastronomy 3 proceedings

Doyle, L.R.-McKay, C.P. (1990) in Bioastronomy 3 proceedings

Drake, F. (1961) Physics Today 14, 40.

Grin, E.A. (1990) in Bioastronomy 3 proceedings

Hart, M.H. (1978) Icarus 39, 23-39.

Herbst, E. (1990) in Bioastronomy 3 proceedings

Irvine, W.M. et al (1980) Nature 283, 748-749.

Irvine, W.M. (1989) Highlights of Astronomy 8, 339-344.

Kasting J.F. (1988) Icarus 74, 472-494.

Levasseur-Regourd, A.-C. (1988), Journal de Physique C1 Suppl.3, 49, Mars 25-35.

Lovelock, J. (1989) "The Ages of Gaia", Oxford University Press.

Marochnik, L.S.-Mukhin, L.M. (1987) in Bioastronomy 2 proceedings 49-60.

Marx, G. (1990)in Bioastronomy 3 proceedings

Matthews, C.N.(1990): in "Environmental Evolution", edited by L.Margulis, M.I.T. Press Maurette, M.-Bonny, Ph. (1990) in Bioastronomy 3 proceedings

Muller, R. (1984) in Bioastronomy 1 proceedings 233-244.

Nussinov, M.D.-Maron, V.I. (1990), Journal of British Interplanetary Society 49, 3-10

Pollack, J.B. et al (1987) Icarus 71, 203-204.

Reynolds, R.T. et al (1987) in Bioastronomy 2 proceedings 21-28. Icarus 56, 246-254

Sagan, C.-Mullen, G. (1972) Science 177, 52.

Squires, S.W. (1989) Icarus 79, 219-228.

Szegö, K. (1987) in Bioastronomy 2 proceedings 31-38.

Teriile, R. (1987) in Bioastronomy 2 proceedings 125-130.

Terille, R. (1990) in Bioastronomy 3 proceedings 